MATHEMATICS OF COMPUTATION

Volume 72, Number 244, Pages 2043-2057

S 0025-5718(03)01514-X

Article electronically published on March 26, 2003

\title{
ON THE UNKNOTTING NUMBER OF MINIMAL DIAGRAMS
}

\author{
A. STOIMENOW
}

Abstract. Answering negatively a question of Bleiler, we give examples of knots where the difference between minimal and maximal unknotting number of minimal crossing number diagrams grows beyond any extent.

\section{INTRODUCTION}

It is known that a diagram $D$ of a knot can be made into a diagram of the unknot by crossing changes. The unknotting number $u(D)$ of $D$ is defined as the minimal number of such crossing changes, and the unknotting number $u(K)$ of a knot $K$ is given by

$$
u(K)=\min _{D \text { diagram of } K} u(D) .
$$

Taking the minimum over an infinite number of diagrams makes the unknotting number of a knot often hard to calculate (see $[\mathrm{KM}] \mathrm{Tr}]$ ). Thus one is led to consider modifications of this definition where only finitely many diagrams are considered. The most appealing idea is to consider just diagrams $D$ of crossing number $c(D)$ equal to the (minimal) crossing number $c(K)$ for $K$. Thus define

$$
u_{\min }(K)=\underset{\substack{D \text { diagram of } K \\ \text { with } c(D)=c(K)}}{\min } u(D) \text { and } u_{\max }(K)=\underset{\substack{D \text { diagram of } K \\ \text { with } c(D)=c(K)}}{\max } u(D) .
$$

We have then for any knot $K$ the obvious inequalities

$$
u(K) \leq u_{\min }(K) \leq u_{\max }(K) .
$$

Suggestively, for many knots both inequalities are in fact equalities. The second inequality holds for alternating knots by the proof of the Tait crossing number $\mathrm{Ka}, \mathrm{Mu}, \mathrm{Th}$ and flyping conjectures $[\mathrm{MT}$, and the first inequality is true for a large class of positive braid knots (including the torus knots) by the work of Murasugi [Mu2, Boileau-Weber [BW], and the proof of the (local) Thom conjecture [KMr].

It was surprising, when Bleiler $[\mathrm{Bl}$ and Nakanishi $\mathrm{Na}$ (independently) found an example, $10_{8}$ in the tables of [Ro, appendix], for which the first inequality was strict $\left(u=2\right.$, but $\left.u_{\min }=3\right)$. Bleiler asked whether there are also examples of knots for which the second inequality is strict. This was confirmed by two examples from Thistlethwaite's tabulations [HTW] given in [St with $u=u_{\min }=1$ but $u_{\max }>1$.

Received by the editor June 6, 2000 and, in revised form, March 8, 2002.

2000 Mathematics Subject Classification. Primary 57M25.

Key words and phrases. Kauffman polynomial, HOMFLY polynomial, crossing number, homogeneous braid, unknotting number.

The author was supported by a DFG grant. 
Bleiler asked furthermore whether there is some (upper) bound on the difference of the hand-sides of both inequalities. While a negative answer to this question for the first inequality follows directly from Bleiler's example and the proof of the Tait conjectures (see below), in this note we will mainly focus on finding examples, using the program KnotScape [HT], answering the question negatively for the second inequality. These examples come from computational results obtained by the tools of Thistlethwaite and myself. More specifically, we have

Theorem 1.1. The number of fibered knots of crossing number at most $15 n$ with $u_{\max }-u_{\min } \geq n$ is asymptotically at least $O\left(n^{587}\right)$.

The easiest way to show, using Bleiler's example, that $u_{\min }-u$ can grow unboundedly is to consider the iterated connected sums of his knot with itself. It is known from $[\mathrm{Ka}, \mathrm{Mu}, \mathrm{Th}$ and [Me that a minimal crossing number diagram of a (possibly composite) alternating knot is the (possibly trivial) connected sum of alternating prime knot diagrams. Thus consider $K_{n}=\#^{n} 10_{8}$. (Here '\#' means connected sum and ' $\#^{n} K$ ' means the connected sum of $n$ copies of $K$.) While $u\left(K_{n}\right)=2 n$ follows from the signature, we have by the above fact $u_{\min }\left(K_{n}\right)=3 n$.

Such examples are rather easy to come by. To obtain a more interesting result, for both inequalities in (1) we will construct prime examples of the desired types (for which less computational and more mathematical arguments are needed).

\section{Conventions and definitions.}

Link polynomials. For an account on the various link polynomials we refer to the papers $\mathrm{LM}] \mathrm{Ka}$.

The skein polynomial $P$ is a Laurent polynomial in two variables $l$ and $m$ of oriented knots and links which is equal to 1 on the unknot, and is defined by the (skein) relation

$$
l^{-1} P(\nearrow)+l P(\searrow)=-m P()()
$$

The Kauffman polynomial is usually defined via a regular isotopy invariant $\Lambda(a, z)$ of unoriented links with the properties

$$
\begin{aligned}
& \Lambda(\nearrow)+\Lambda(\lambda)=z(\Lambda(\stackrel{\sim}{\sim})+\Lambda()()) \\
& \Lambda(\curlywedge \bigcirc)=a \Lambda(\mid) ; \quad \Lambda(\curlywedge \bigcirc)=a^{-1} \Lambda(\mid), \\
& \Lambda(\bigcirc)=1
\end{aligned}
$$

The Kauffman polynomial $F(D)$ of a link represented by an oriented diagram $D$ is then defined as $a^{-w(D)} \Lambda(a, z)$, where $w(D)$ is the writhe of $D$.

The Jones polynomial $V$, Brandt-Lickorish-Millett-Ho polynomial $Q$ and (one variable) Alexander polynomial $\Delta$ are obtained from $P$ and $F$ by the substitutions

$$
\begin{aligned}
V(t) & =P\left(-i t, i\left(t^{-1 / 2}-t^{1 / 2}\right)\right)=F\left(-t^{-3 / 4}, t^{1 / 4}+t^{-1 / 4}\right), \\
\Delta(t) & =P\left(i, i\left(t^{1 / 2}-t^{-1 / 2}\right)\right) \\
Q(z) & =F(1, z) .
\end{aligned}
$$

For $P$ and $F$ there are several other conventions of variables, differing from each other by possible inversion and/or multiplication of some variable by some fourth root of unity. However, our arguments apply regardless of the convention used. We 


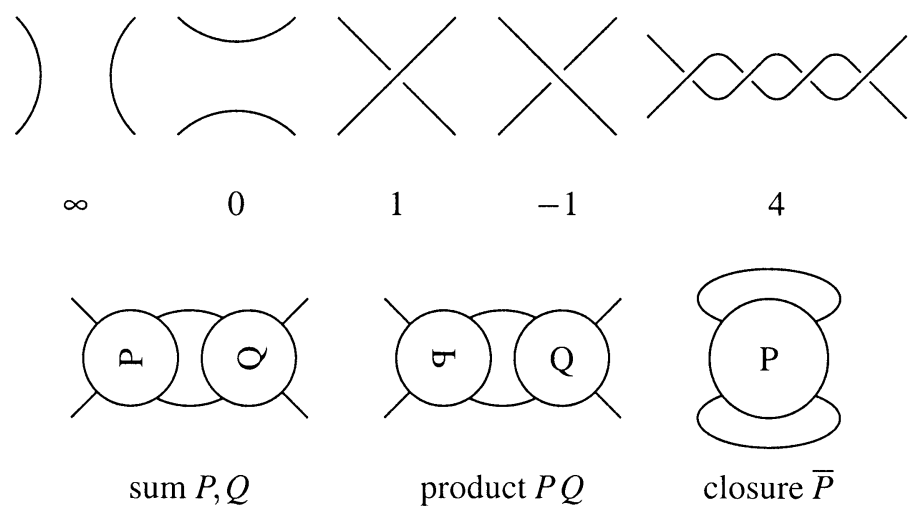

Figure 1. Conway's tangles and operations with them. (The designation 'product' is very unlucky, as this operation is neither commutative, nor associative, nor is it distributive with 'sum'.)

will only assume the invariants to be normalized so that the unknot has polynomial 1.

We will sometimes write $V_{D}, \Delta_{D}, \ldots$ for $V(D), \Delta(D), \ldots$, etc. Also, we will not notationally distinguish arguments to the polynomials, which are variables/numbers, diagrams, or links, since the meaning of the expression will not be ambiguous.

Let $[Y]_{t^{a}}=[Y]_{a}$ be the coefficient of $t^{a}$ in a polynomial $Y \in \mathbb{Z}\left[t^{ \pm 1}\right]$. Let the minimal and maximal degree and span (or breadth) of $Y \neq 0$ be given by

$$
\begin{gathered}
\min \operatorname{deg} Y=\min \left\{a \in \mathbb{Z}:[Y]_{a} \neq 0\right\}, \max \operatorname{deg} Y=\max \left\{a \in \mathbb{Z}:[Y]_{a} \neq 0\right\}, \\
\operatorname{span} Y=\max \operatorname{deg} Y-\min \operatorname{deg} Y .
\end{gathered}
$$

For $Y \in \mathbb{Z}\left[x_{1}, \ldots, x_{n}\right]$ and some monomial $X$ in the $x_{i}$, we denote by $[Y]_{X}$ the coefficient of $X$ in $Y$. max cf $Y$ denotes the leading coefficient of $Y$, i.e., $[Y]_{\max } \operatorname{deg} Y$.

Knot notation. The knots of $\geq 11$ crossings occurring below are always identified according to their numbering in the tables of KnotScape [HT]. They can be loaded and drawn by this program, if so desired. The knots of $\leq 10$ crossings are identified according to the tables of [ $\underline{\mathrm{RO}}$, appendix].

The obverse (mirror image) of $K$ is denoted by $! K$.

Algebraic tangles and Conway notation. We briefly introduce the algebraic tangles which will be applied in the subsequent constructions.

Algebraic tangles were introduced by Conway [Co]. These are the tangles that can be built up from the primitive tangles 0,1 and $\infty$ by iterated application of tangle sum and product, as shown in Figure 1 See [Ad, §2.3] for example.

\section{EXAMPles OF ADEQUATE KNOTS}

The most naive idea for dealing with the second inequality in (1) is to adapt the argument used above for the first inequality: take iterated connected sums of an appropriate knot $K$ having $u_{\min }<u_{\max }$. The problem is, however, that we do not know in general how to show that $c\left(\#^{n} K\right)=n c(K)$. The arguments based on the 


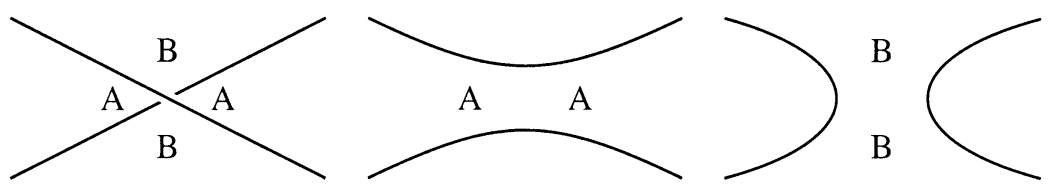

Figure 2. The $A$ - and $B$-corners of a crossing, and its both splicings. The corner $A$ (resp. $B$ ) is the one passed by the overcrossing strand when rotated counterclockwise (resp. clockwise) towards the undercrossing strand. A type $A$ (resp. $B$ ) splicing is obtained by connecting the $A$ (resp. $B$ ) corners of the crossing.

span of the Jones [Ka, $\mathrm{Mu}, \mathrm{Th}]$ and $Q$ [Ki] polynomials work only for alternating knots, for which, as remarked, the Tait conjectures imply $u_{\min }=u_{\max }$.

One possible way to circumvent this problem is to turn back to Thistlethwaite's inequalities for the Kauffman polynomial $F$ [Th2]. He showed that, if $\Lambda$ denotes the (writhe) unnormalized version of $F$, the coefficient $[\Lambda(D)]_{a^{k} z^{l}}$ of the monomial $a^{k} z^{l}$ in $\Lambda(D)$ for a given diagram $D$ is nonzero only if $|k|+l \leq c(D)$. This fact poses restrictions on the writhe of diagrams of given crossing number with given $F$ polynomial. We are interested in cases when these restrictions determine the writhe uniquely.

In [Th3] these cases were described by a diagrammatic condition related to Kauffman's state model Ka2].

Definition 2.1 (see [Th3]). A splicing of a crossing in an (unoriented) link diagram $D$ is a local move replacing the leftmost diagram in Figure 2 by the middle $(A$ splicing) or rightmost ( $B$-splicing) diagram. The choice of (one of the two) splicings for each crossing in $D$ is called a state of $D$. Performing the splicings chosen in $S$ reduces $D$ to a collection of disjoint loops. The $A$-state of the diagram is the state in which all crossings are $A$-spliced. Similarly the $B$-state is the state in which all crossings are $B$-spliced. A diagram is called $A$-adequate, if changing the splicing of any crossing (from $A$ to $B$ ) in the $A$-state reduces (by 1 ) the resulting number of loops. Analogously one defines $B$-adequacy, and calls $D$ adequate, if it is both $A$-adequate and $B$-adequate. A knot $K$ is adequate, if it has an adequate diagram $D$.

Theorem 2.1 ([Th3, Theorem 3(i)]). A knot diagram $D$ is adequate if and only if

$$
\max _{[F(D)]_{a_{z} l} \neq 0} k+l-c(D)=\min _{[F(D)]_{a^{k} z^{l}} \neq 0} k-l+c(D) .
$$

The following consequences, noted in [Th3, are immediate from the above result.

Proposition 2.1. If a knot $K$ has an adequate diagram $D$, then $c(K)=c(D)$, and moreover, all minimal crossing number diagrams of $K$ have the same writhe as $D$.

Corollary 2.1. If $K_{1,2}$ are adequate knots, then $c\left(K_{1} \# K_{2}\right)=c\left(K_{1}\right)+c\left(K_{2}\right)$.

It turns out that, for the purpose of evaluating knot tables, it is easier to test adequacy by its defining property in Definition 2.1 than by the property given in Theorem 2.1] Thus, using Definition [2.1, we have a convenient method for selecting knots from the table, for some of which we need to show that $u_{\min }<u_{\max }$.

It turned out that there were no appropriate examples with $u_{\max }>1=u_{\min }$, so we were led to consider the next simplest case $u_{\max }>2 \geq u_{\min }$. This means 
we seek knots for which there are minimal crossing number diagrams which are unknotted by 2 crossing changes, and others that are not.

Using Thistlethwaite's tools, one can generate many diagrams of the same knot by applying a variety of moves on an input diagram. For these diagrams the unknotting number can be determined by switching crossings and testing the result according to triviality of the Jones polynomial. (This gives a very effective knottedness test at reasonable crossing numbers.)

This procedure, when given as input the about nineteen thousand $(18,982)$ adequate nonalternating prime knots of $\leq 15$ crossings (in their diagrams included in the table), gave a list of 57 knotg 1 with $u_{\max }>2 \geq u_{\min }$. Thus all possible (including mixed) iterated connected sums of such knots give the desired examples.

There are 5 knots $K$ among these $57\left(14_{25034}, 14_{25151}, 14_{25154}, 14_{25758}\right.$ and $\left.14_{25835}\right)$ whose double (branched) cover $D_{K}$ has noncyclic (integral) homology group $H_{1}\left(D_{K}\right)$ (for the first three it is $\mathbb{Z}_{9} \oplus \mathbb{Z}_{9}$, and for the last two $\mathbb{Z}_{45} \oplus \mathbb{Z}_{3}$ ), and hence by [We] we have $u\left(\#^{n} K\right)=n u(K)=2 n$.

For all the remaining 52 examples, for which $H_{1}\left(D_{K}\right)$ is cyclic, I could not decide whether $u\left(\#^{n} K\right)=n u(K)$. None of them had signature \pm 4 , and I could show $u(K)=2$ only for 12 further examples. First, for three of them, $15_{112110}, 15_{113676}$ and $15_{113679}$, I could apply the (somewhat sophisticated) method of Traczyk [Tr] (working for $10_{67}$ and involving a combination of the signature and the LickorishMillett value $\left.V\left(e^{\pi i / 3}\right)\right)$. The other 9 knots I could deal with by the criterion of Lickorish [Li].

\section{Homogeneous BRAid eXAmples}

An alternative way to construct examples with $u_{\max }>u_{\min }$ is to use Murasugi's results on homogeneous braid knots [Mu2, Proposition 7.4]. Homogeneous braids were introduced by Stallings $\left[\mathrm{S}\right.$ ] and are braids in which each Artin generator $\sigma_{i}$ appears entirely with positive or entirely with negative powers. The quest for such examples appeared to be the more difficult alternative compared to adequate knots, when using Thistlethwaite's tabulations. The property to be the closure of a homogeneous braid of minimal strand number is not straightforward to test, and even the (larger) set of knots obtained by testing the necessary conditions on the HOMFLY polynomial from [MP] is relatively small.

Thus (closures of) homogeneous braids to which the Murasugi results apply are much less common among $\leq 15$ crossing prime knots than adequate knots. Nonetheless, there were surprisingly more examples of this type, as it turns out that the phenomenon $u_{\max }>u_{\min }$ occurs much more frequently. (This outcome may be partially explained by the extension of the flyping results for alternating links to certain classes of adequate links, as shown in [Th4].) Here we describe how we obtained these examples, giving a proof of Theorem 1.1

Proof of Theorem 1.1. Murasugi's idea to estimate $c(D)$ for a diagram $D$ of a knot $K$ is to write

$$
c(D)=(c(D)-s(D)+1)+(s(D)-1)
$$

\footnotetext{
${ }^{1}$ Although Thistlethwaite's tools use a rich variety of moves, for some few special knots not all minimal crossing number diagrams are interconvertible by these moves, so there may be some (but really very few, if any) more knots we could have missed.
} 
with $s(D)$ being the number of Seifert circles of $D$, and to estimate both parts from the HOMFLY polynomial separately. For the first part we have from [Mo]

$$
c(D)-s(D)+1 \geq \max \operatorname{deg}_{m} P(K) .
$$

For a homogeneous braid diagram $D$ this inequality is sharp (see the genus results in $[\mathrm{Cr}]$ ), and $s(D)$ is the number of strands of the braid. Thus the homogeneous braid diagram $D$ is of minimal crossing number if the braid representation is of minimal strand number. (Beware that the converse is not true; see St3.)

The handiest way to replace the (strand number) minimality condition of the homogeneous braid is to use the (sharpness of the) MWF inequality [Mo, FW]:

$$
s(D) \geq \frac{1}{2} \operatorname{span}_{l} P(K)+1 .
$$

(Other methods to exhibit this minimality are rather elaborate and available only for small strand number, see, e.g., $\mathrm{K}$, MS.)

Thus we are interested in knots $K$ for which $\operatorname{span}_{l} P_{K}+2 \max \operatorname{deg}_{m} P_{K}=2 c(K)$ and $\max \operatorname{cf}_{m} P_{K}=l^{k}$ for some $k \in 2 \mathbb{Z}$, where for $P$ we use the convention of [LM]. The second property comes from $[\mathrm{MP}$, as (closures of) homogeneous braids are *-products (Murasugi sums) of $(2, n)$-torus links.

The resulting conditions were satisfied by (the $P$ polynomials of) 10,507 of the nonalternating $\leq 15$ crossing prime knots. 371 of them passed the test for $u_{\max }>$ $2 \geq u_{\text {min }}$. From these knots, 160 could be exhibited to have homogeneous braid diagrams (by testing the property on the diagrams generated by Thistlethwaite's tools).

Calculating the symmetry group of the 160 knots with the tool provided in KnotScape (a version of Jeff Weeks's hyperbolic symmetry program), I found that all except 28 had no symmetries, while these 28 knots had symmetry group $\mathbb{Z}_{2}$. For 25 of the knots the nontrivial symmetry is an inversion, for one other knot, $14_{36031}$, a simultaneous in- and obversion (i.e., the knot is -achiral), while for the remaining two knots, $14_{34103}$ and $15_{201628}$, it preserves longitude and meridian (and so all the four in- and obverted versions are distinct). Thus, together with their inand obversed versions, the 160 examples give $4 \cdot 160-2 \cdot 26=588$ knots, which can be used to build connected sums. This way we arrive at the statement given in Theorem 1.1.

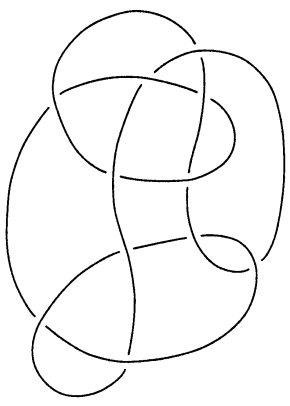

$11_{408}$

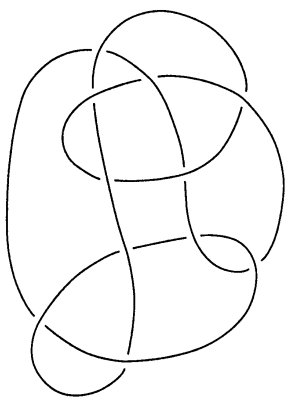

$11_{414}$

Figure 3. 
Furthermore, 89 among these 160 knots have signature \pm 4 , so that their iterated (mixed) connected sums (with properly obversed factors) can be shown to have $u=$ $u_{\text {min }}$. Among these 89 knots are also the two simplest examples in the list of 160 , the mutants $11_{408}$ and $11_{414}$, see Figure 3. (They have the 4-braid representations $\sigma_{1} \sigma_{2}^{3} \sigma_{3}^{-1} \sigma_{1} \sigma_{2}^{2} \sigma_{3}^{-1} \sigma_{2} \sigma_{3}^{-1}$ and $\left(\sigma_{1} \sigma_{2}^{2} \sigma_{3}^{-1}\right)^{2} \sigma_{2} \sigma_{1} \sigma_{3}^{-1}$ respectively.)

\section{Prime examples}

More interestingly, it is possible to construct a series of prime knots with the previous properties. This can be achieved by modifying the connected sums of the above examples and trying to preserve the necessary conditions. The arguments for the proof this time are mathematical rather than computational, albeit they make decisive use of the specific examples found above. It is most appropriate to focus on a single such example in the proof.

Theorem 4.1. For any $n \in \mathbb{N}$, there exist prime knots $K_{n}$ with $u\left(K_{n}\right)=u_{\min }\left(K_{n}\right)$ and $u_{\max }\left(K_{n}\right)-u_{\min }\left(K_{n}\right) \rightarrow \infty$.

Proof. Consider the tangle $T$ given by the 2 diagrams in Figure 4. (It is an easy exercise to see that both diagrams represent the same tangle.)

$D_{1}$ and $D_{2}$ were obtained by cutting off two diagrams $D_{1}^{\prime}$ and $D_{2}^{\prime}$ of one of the knots mentioned above, $14_{25034}$. Thus $D_{i}^{\prime}$ is the closure of $D_{i}, i=1,2$. $D_{1}^{\prime}$ can be unknotted by two crossing changes, but $D_{2}^{\prime}$ cannot. (This was tested by switching crossings and calculation of the Jones polynomial.)

Consider the knot

$$
K_{n}=(\underbrace{T, T, \ldots, T}_{n \text { copies }}), 3,-3
$$

in its diagrams

$$
\hat{D}_{1, n}=(\underbrace{D_{1}, \ldots, D_{1}}_{n \text { copies }}), 3,-3
$$

and

$$
\hat{D}_{2, n}=(\underbrace{D_{2}, \ldots, D_{2}}_{n \text { copies }}), 3,-3 .
$$

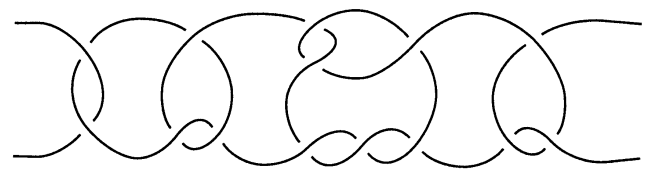

$D_{1}$

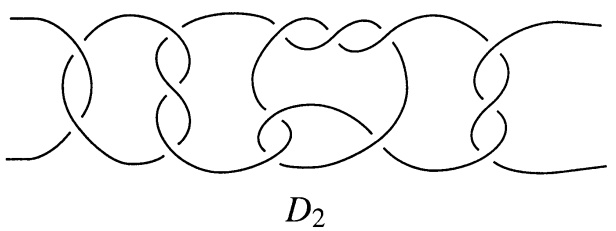

FIGURE 4. 
(Here ',' is the Conway tangle sum operator, and ' \pm 3 ' denote the primitive Conway tangles, see Figure 1)

$K_{n}$ is arborescent, and hence prime. This can also be shown by first showing that $T$ is prime by repeated application of the result of [Va], classifying primeness of Conway sum of tangles, and then using the argument of [KL].

Now, the $A$ and $B$ splicings $D_{2}$ both yield a collection of loops and an $\infty$-tangle (rather than a 0-tangle). Switching the splicing of one of the crossings either joins two of these loops, or it joins one loop to one of the components of the $\infty$-tangle. From this one can see that the diagram $\hat{D}_{2, n}$ is adequate, hence of minimal crossing number, and that hence so is $\hat{D}_{1, n}$.

$\hat{D}_{1, n}$ differs from $\#^{n} D_{1}$ just by 2 crossing changes, and hence $u\left(\hat{D}_{1, n}\right) \leq 2 n+2$. In fact, we show that $\hat{D}_{1, n}$ realizes the unknotting number of $K_{n}$.

By 3-moves

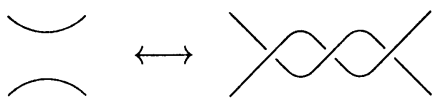

and isotopies, $T$ can be transformed into the 0 -tangle with 2 additional loops. Thus $K_{n}$ can be transformed by 3 -moves into $2 n+3$ loops. As this number of loops is known to be $\operatorname{dim}_{\mathbb{Z}_{3}} H_{1}\left(D_{K_{n}}, \mathbb{Z}_{3}\right)+1$, and by [We] $u\left(K_{n}\right) \geq \operatorname{dim}_{\mathbb{Z}_{3}} H_{1}\left(D_{K_{n}}, \mathbb{Z}_{3}\right)$, we obtain $u\left(K_{n}\right) \geq 2 n+2$ (see St2]).

Contrarily, we must show $u\left(\hat{D}_{2, n}\right)-2 n \rightarrow \infty$. We make a computer calculation of what combinations of determinants and signatures $\sigma$ occur for diagrams, obtained by switching at most 2 crossings in $D_{2}^{\prime}$, in case the determinant is not divisible by 3. The list is as follows:

\begin{tabular}{c||c|c|c|c|c|c}
$\operatorname{det}$ & 11 & 13 & 17 & 19 & 23 & 53 \\
\hline$\sigma$ & 2 & -4 & 0 & -2 & -2 & 0
\end{tabular}

Now assume that $u\left(\hat{D}_{2, n}\right)-2 n$ is bounded when $n \rightarrow \infty$. Fix in each diagram $\hat{D}_{2, n}$ a minimal set $S_{n}$ of crossings unknotting the diagram. Denote by $F_{n}$ the (unknot) diagram obtained from $\hat{D}_{2, n}$ after switching the crossings in $S_{n}$. Let $c_{n, i}$ be the number of crossings in $S_{n}$ which lie in the $i$-th copy of $D_{2}$ in $\hat{D}_{2, n}$, and let $E_{n, i}$ be the diagram obtained by closing up this $i$-th copy, with those $c_{n, i}$ crossings switched.

We have that the (un)knot on $F_{n}$ differs by at most two crossing changes from the knot $L_{n}$ represented by the diagram $\#_{i=1}^{n} E_{n, i}$; thus this knot has unknotting number $\leq 2$ for all $n$.

Now,

$$
\left|\sum_{i=1}^{n} c_{n, i}-u\left(\hat{D}_{2, n}\right)\right| \leq 6
$$

follows directly from the definition of $c_{n, i}$, so that

$$
\left|\sum_{i=1}^{n} c_{n, i}-2 n\right|
$$

must be bounded. This means that

$$
k_{n}:=\#\left\{1 \leq i \leq n: c_{n, i}<3\right\}
$$

grows unboundedly for $n \rightarrow \infty$. 
Consider the diagrams $E_{n, i}$ with $c_{n, i}<3$, and number them $E_{n, i_{1}}, \ldots, E_{n, i_{k_{n}}}$. For any prime

$$
p \in \mathcal{P}:=\{3,11,13,17,19,23,53\},
$$

let $l_{n, p}$ be the number of occurrences of $p$ as divisor of the determinant of the (knots represented by the) $k_{n}$ diagrams $E_{n, i_{1}}, \ldots, E_{n, i_{k_{n}}}$, with multiple divisors $p$ of a single determinant counted as only one occurrence. That is,

$$
l_{n, p}:=\#\left\{1 \leq j \leq k_{n}: p \mid \operatorname{det}\left(E_{n, i_{j}}\right)\right\} .
$$

Then

$$
\sum_{p \in \mathcal{P}} l_{n, p} \geq k_{n}
$$

For fixed $n$, choose $p \in \mathcal{P}$ such that $l_{n, p}$ is maximal, and set $p_{n}=p$ and $l_{n}=l_{n, p_{n}}$. Then $l_{n} \geq k_{n} / 7$. But then by [We] we have

$$
u\left(L_{n}\right) \geq \operatorname{dim}_{\mathbb{Z}_{p_{n}}} H_{1}\left(D_{L_{n}}, \mathbb{Z}_{p_{n}}\right)=l_{n} \geq k_{n} / 7 \longrightarrow \infty,
$$

a contradiction to the above condition that $u\left(L_{n}\right) \leq 2$.

This contradiction shows that $\left\{s_{n}\right\}:=\left\{u\left(\hat{D}_{2, n}\right)-2 n\right\}$ has an unbounded subsequence, but arguing with any subsequence of $\left\{s_{n}\right\}$ instead shows that $\left\{s_{n}\right\}$ is itself unbounded. (Of course, one can also give an explicit estimate for $u\left(\hat{D}_{2, n}\right)$, for which one would more economically consider just $p_{n} \in\{3,17,53\}$ and use $\sigma$.)

Similar methods should be applicable to some of the other computer examples given above. First one needs to find adequate diagrams $D_{1,2}$ of the knot of different unknotting numbers $u_{1}<u_{2}$, such that $D_{2}$ cannot be made by $\leq u_{1}$ crossing changes into a diagram of a knot with $\sigma=0$ and det $=1$. Then one needs to exhibit $D_{1,2}$ to be closures of different diagrams of the same tangle $T$, whose $A$ and $B$-states both connect its endpoints as in the tangle $\infty$ (i.e., not as in the 0 -tangle), and then to argue why $K_{n}$ is prime (e.g., by arborescence, or because $T$ is prime). In order to distinguish the examples, one should pick up diagrams $D_{1,2}$ of knots $K$ with different values of $V_{K}(t) V_{K}(1 / t)$ and choose $p$ odd so that the values of $V_{K}\left(e^{\pi i / p}\right)$ are also all of different norm on these diagrams. Then to build $\hat{D}_{1,2}$, instead of the $(3,-3)$-tangle one uses the $(p,-p)$-tangle, as this preserves $\left|V\left(e^{\pi i / p}\right)\right|$.

The method of the last proof can be used to show an analogous statement for the first inequality in (1).

Theorem 4.2. For any $n \in \mathbb{N}$, there exist prime knots $K_{n}$ with $u_{\max }\left(K_{n}\right)=$ $u_{\min }\left(K_{n}\right)$ and $u_{\min }\left(K_{n}\right)-u\left(K_{n}\right) \rightarrow \infty$.

Proof. Since the argument is a modification of the one in the last proof, we just indicate how to alter the details.

As $T$ take the rational tangle of Bleiler's knot, 29/6, in its two diagrams considered by Bleiler, $D_{1}=2-22-224$ and $D_{2}=514$. Take

$$
K_{n}=(\underbrace{T, T, \ldots, T}_{n \text { copies }}), 3,3
$$

(i.e., replace the terminal ' -3 ' in the last proof by a ' 3 '). Then $K_{n}$ is alternating. Its primeness follows by the same arguments as above, or more directly from $\mathrm{Me}$. As before, $\hat{D}_{1, n}$ (defined as above, but again with ' -3 ' replaced by ' 3 ') shows 
that $u\left(K_{n}\right) \leq 2 n+3$. By [MT], alternation implies also $u_{\min }\left(K_{n}\right)=u_{\max }\left(K_{n}\right)$, and all minimal diagrams of $K_{n}$ have the same unknotting number. Therefore, to estimate $u_{\min }\left(K_{n}\right)$ (and to show that $u_{\min }\left(K_{n}\right)-2 n \rightarrow \infty$ ), it suffices to consider a particular alternating diagram of $K_{n}$. Consider $\hat{D}_{2, n}$. Again use that all ways of switching two crossings in $D_{2}$ give tangles, which close to knots with nonunit determinants. (All possibilities have been explicitly listed up by Bleiler in [B], but we can argue more directly because all these tangles are rational and nontrivial.) Then the argument in the previous proof gives the same contradiction under the assumption that $u_{\min }\left(K_{n}\right)-2 n$ is bounded.

\section{Related RESUlts AND QUESTIONS}

The method applied above also yields the following statements.

Theorem 5.1. For any $n \geq 11$ there is a knot of $n$ crossings with unit Alexander polynomial, except possibly for $n \in\{17,18,19,20,21,23\}$.

Theorem 5.2. For any $n \geq 10$ there is a fibered knot of $n$ crossings with unit determinant, except possibly for $n \in\{17,18,19,21,23\}$.

(The exceptions for $n$ in both theorems are unlikely, albeit I cannot definitely exclude them.)

Proof. For both assertions one takes connected sums of adequate (Theorem 5.1) or homogeneous braid knots of Murasugi type (Theorem 5.2). For $n=11$ in Theorem 5.2 we have no such knots, but the two determinant one knots $11_{416}$ and $11_{483}$ are fibered (and of genus two), which can be shown by Gabai's method [Ga]. (The other two knots with unit determinant are those with unit Alexander polynomial, which are clearly not fibered.)

Again, with more effort one can try to construct prime knots. We present the construction for Theorem 5.1 (I do not know how to do this for Theorem[5.2), in a slightly weaker version, again using more specific examples.

Theorem 5.3. For almost all numbers $c \in \mathbb{N}$ there is a prime knot $K$ with crossing number $c$ and unit Alexander polynomial.

Before we start with the proof, we make two definitions.

Definition 5.1. From the 2 splicings of a crossing in a knot diagram, we call the one giving a two component link the skein splicing and the other one, giving a knot, the Kauffman splicing.

Definition 5.2. For a tangle



we call

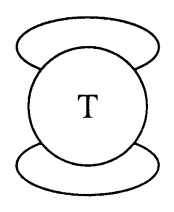


the numerator closure of $T$ and

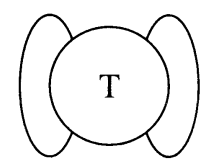

the denominator closure of $T$.

Proof of Theorem 5.3. As before, we have to modify the connected sums into a prime knot preserving the Alexander polynomial. How to do this was shown by Bleiler [Bl2], but we need to refine his method so as to keep track of adequacy.

Consider a knot diagram of the type

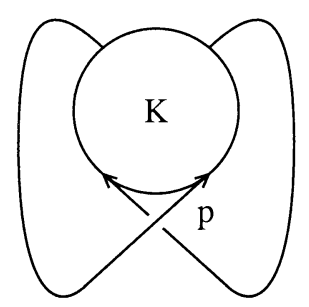

We can produce such a picture (with appropriate tangle $K$ ) each time we are given a knot diagram $D$ and a distinguished crossing $p$. In this case we write $K=K(D, p)$ to indicate from which diagram $D$ and crossing $p$ we obtained $K$. (The choice of sign of $p$ here is irrelevant; the argument goes through also with the mirrored diagrams.) Note that $K$ has the homotopy type

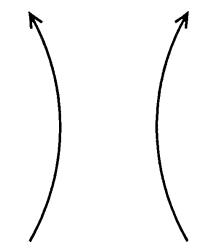

Define $T$ to be the tangle

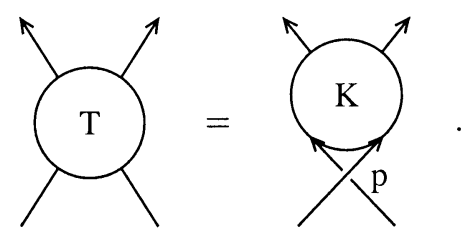

We again write $T(D, p)$ to indicate from which $D$ and $p$ the tangle $T$ originates, if needed. By $T^{\prime}$ we denote either $T$ with the orientation of both components reversed or $T$ flipped by $180^{\circ}$ around the horizontal axis of the projection plane. (These two operations give in general different results, but our arguments apply to both in the same way.) 
We will consider a diagram of the type

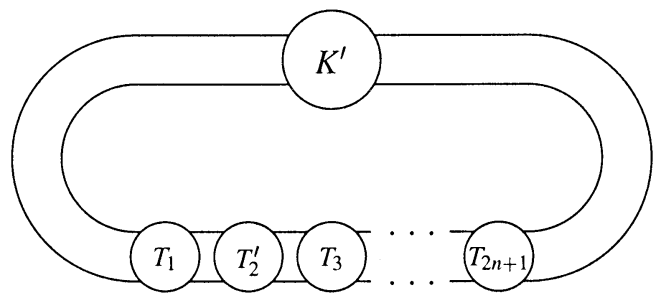

and would like to choose the tangles $T_{i}$ and $K$ so that (4) becomes a minimal crossing number diagram of a prime knot with $\Delta=1$.

First we choose the $T_{i}$ so that they are prime and their denominator closure is a $\Delta=1$ knot. By the proof of Lemma 2.1 of [Bl2], to ensure primeness it suffices that both strands of $T_{i}$ be unknotted and the numerator closure of $T$ not be a rational knot. If $T_{i}$ was obtained in the above way from a knot diagram $D_{i}$ with a distinguished crossing $p_{i}$, then these conditions mean that the skein splicing of $p_{i}$ in $D_{i}$ gives a two-component link with both components unknotted, and the Kauffman splicing of $p_{i}$ gives a nonrational knot. The same conditions also ensure the primeness of $K\left(D_{i}, p_{i}\right)$.

By an argument analogous to that of Bleiler with the "KT-grabber" (looking at the Alexander polynomials of both closures of the tangle) we see that a replacement

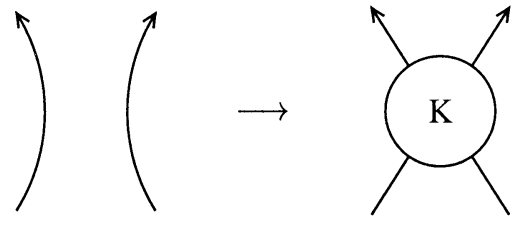

preserves $\Delta$, if both

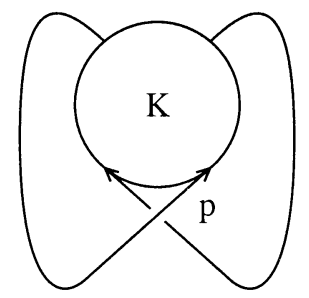

and

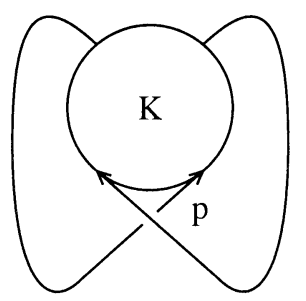

have $\Delta=1$. Thus to find an appropriate tangle $K$ as $K(D, p)$ for proper $D$ and $p$ we need, additionally to the above two primeness conditions and the condition that $\Delta(D)=1$, the condition that switching $p$ in $D$ preserves $\Delta=1 . T$ is then defined as in (3)).

Consider the following two diagrams with distinguished crossings, found with partial computer help and shown in Figure 5.

Both diagrams depict minimal crossing number diagrams of adequate $\Delta=1$ knots. In both cases the skein splicing of the distinguished crossing gives a link of 2 unknots and the Kauffman splicing gives a nonrational (in fact, nonalternating) knot. (On the left it is $13_{8775}$, which also has $\Delta=1$, and on the right $9_{44}$.) Moreover, switching $p_{1}$ in $D_{1}$ gives the knot $11_{401}$ on the right, so $\Delta=1$ is preserved. 


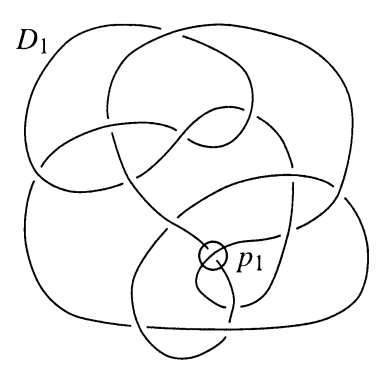

$14_{30599}$



$11_{401}$

FiguRe 5 .

Thus for any choice of $T_{i} \in\left\{\hat{T}_{1}, \hat{T}_{2}\right\}$ with $\hat{T}_{j}=T\left(D_{j}, p_{j}\right), j=1,2$, and $K=$ $K\left(D_{1}, p_{1}\right)$ we have that (4) is a diagram of a $\Delta=1$ knot, which is prime by [KL]. It remains to prove that the diagram is adequate, and hence of minimal crossing number.

First we note that the $\hat{T}_{i}$ are adequate, i.e., in their $A$ - and $B$-state any switch of the state of a crossing reduces the number of free loops or switches the connection of the four tangle ends between the 0 - and $\infty$-tangle.

Moreover, in the $A$ - and $B$-states of both $\hat{T}_{1}$ and $\hat{T}_{2}$ the four endpoints are connected differently (i.e., once by a 0 - and once by an $\infty$-tangle). Without loss of generality, obvert (mirror) $\hat{T}_{i}$ so that the $B$-states have an $\infty$-tangle. (This spoils neither primeness, nor $\Delta=1$.)

Now we use the fact that

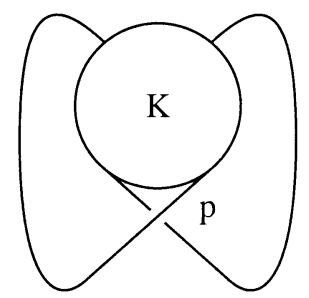

is an adequate diagram.

Consider the $B$-state of (44) and choose a crossing $q$ whose splicing is switched (from $B$ to $A$ ).

Assume first $q$ belongs to one of the $T_{i}$ (or $T_{i}^{\prime}$ ). Since $T_{i}$ is adequate, switching $q$ 's splicing either joins (a) two loops in the $B$-state of $T_{i}$, or (b) one loop with one of the two arcs of the 0 -tangle (remaining from $T_{i}$ in its $B$-state), or (c) the two arcs of this 0 -tangle (thereby giving an $\infty$-tangle). In cases (a) and (b), the number of components is clearly reduced, and this also happens in case (c), because this corresponds to switching the state of $p$ in the $B$-state of the diagram (5), which is adequate.

If $q$ belongs to $K$, then switching $q$ 's state reduces the number of loops again because of the adequacy of (5), and because the $B$-state of the $T_{i}$ 's has connected the endpoints of $K$ in the same way as the $B$-splicing of $p$ in (5) would have done.

The same argument applies for the $A$-splicings. Thus (4) is adequate for any choice of $T_{i} \in\left\{\hat{T}_{1}, \hat{T}_{2}\right\}$ and any $n \in \mathbb{N}$. To show that almost all crossing numbers 
can be realized by (4) this way becomes equivalent to showing that all sufficiently large numbers can be written as the sum of an odd number of summands 11 and 14. This is easy to see. (For example, represent $x-154$ for $x$ large enough as sum of summands 11 and 14 and add a representation of 154 by 11 copies of 14 or 14 copies of 11 to get the total number of summands to be odd.)

We finally conclude by a question.

Question 5.1. Are there (sequences of) knots for which

1) both inequalities in (11) are simultaneously strict, or where stronger

2) the difference of the two sides of both inequalities grows simultaneously beyond any extent, or

3) for some of the inequalities one side is bounded, while the other grows unboundedly?

\section{ACKNOWLEDGMENTS}

The calculations have been performed using the programs and tables provided by Ewing, Millett, Hoste, Thistlethwaite and Weeks in HT (and some self-written code building on them). I would like to express my gratitude to them all for making available these data and tools.

I would also like to thank the referee, M. Kidwell, J. Mighton and M. Thistlethwaite for some helpful remarks, and C. Cerf for sending me a copy of [Co].

\section{REFERENCES}

[Ad] C. C. Adams, Das Knotenbuch, Spektrum Akademischer Verlag, Berlin, 1995 (The knot book, W. H. Freeman \& Co., New York, 1994). MR 94m:57007

[Bl] S. A. Bleiler, A note on unknotting number, Math. Proc. Camb. Phil. Soc. 96 (1984), 469-471. MR 85m:57006

[Bl2] , Realizing concordant polynomials with prime knots, Pacific J. Math. 100(2) (1982), 249-257. MR 84e:57004

[BW] M. Boileau and C. Weber, Le problème de J. Milnor sur le nombre gordien des nœuds algébriques, Enseign. Math. 30 (1984), 173-222. MR 86c:57004

[Co] J. H. Conway, An enumeration of knots and links, in "Computational problems in abstract algebra" (J. Leech, ed.), 329-358, Pergamon Press, 1969. MR 41:2661

[Cr] P. R. Cromwell, Homogeneous links, J. London Math. Soc. (series 2) 39 (1989), 535-552. MR 90f:57001

[FW] J. Franks and R. F. Williams, Braids and the Jones polynomial, Trans. Amer. Math. Soc. 303 (1987), 97-108. MR 88k:57006

[Ga] D. Gabai, Detecting fibred links in $S^{3}$, Comment. Math. Helv. 61(4) (1986), 519-555. MR 88c:57009

$[\mathrm{H}] \quad$ P. Freyd, J. Hoste, W. B. R. Lickorish, K. Millett, A. Ocneanu and D. Yetter, A new polynomial invariant of knots and links, Bull. Amer. Math. Soc. 12 (1985), 239-246. MR 86e:57007

[HT] J. Hoste and M. Thistlethwaite, KnotScape, a knot polynomial calculation program, available at http://www.math.utk.edu/ñorwen.

[HTW] J. Hoste, M. Thistlethwaite, and J. Weeks, The first 1,701,936 knots, Math. Intell. 20 (4) (1998), 33-48. MR 97i:57015

[K] T. Kanenobu, Relations between the Jones and $Q$ polynomials of 2-bridge and 3-braid links, Math. Ann. 285 (1989), 115-124. MR 90i:57002

[KM] and H. Murakami, 2-bridge knots of unknotting number one, Proc. Amer. Math. Soc. 98(3) (1986), 499-502. MR 87i:57005

[Ka] L. H. Kauffman, An invariant of regular isotopy, Trans. Amer. Math. Soc. 318 (1990), 417-471. MR 90g:57007 
[Ka2] State models and the Jones polynomial, Topology 26 (1987), 395-407 MR 88f:57006.

[Ki] M. Kidwell, On the degree of the Brandt-Lickorish-Millett-Ho polynomial of a link, Proc. Amer. Math. Soc. 100 (1987), 755-761. MR 89b:57003

[KL] R. C. Kirby and W. B. R. Lickorish, Prime knots and concordance, Math. Proc. Cambridge Philos. Soc. 86(3) (1979), 437-441. MR 80k:57011

[KMr] P. B. Kronheimer and T. Mrowka, The genus of embedded surfaces in in the projective plane, Math. Res. Lett. 1 (1994), 797-808. MR 96a:57073

[Li] W. B. R. Lickorish, The unknotting number of a classical knot, in "Contemporary Mathematics" 44 (1985), 117-119. MR 87a:57012

[LM] and K. C. Millett, A polynomial invariant for oriented links, Topology 26 (1) (1987), 107-141. MR 88b:57012

[Me] W. W. Menasco, Closed incompressible surfaces in alternating knot and link complements, Topology 23 (1) (1984), 37-44. MR 86b:57004

[MT] _ and M. B. Thistlethwaite, The Tait flyping conjecture, Bull. Amer. Math. Soc. 25 (2) (1991), 403-412. MR 92b:57017

[Mo] H. R. Morton, Seifert circles and knot polynomials, Proc. Camb. Phil. Soc. 99 (1986), 107-109. MR 87c:57006

[MS] and H. Short, The 2-variable polynomial of cable knots, Math. Proc. Camb. Philos. Soc. 101 (1987), 267-278. MR 88f:57009

$[\mathrm{Mu}] \quad$ K. Murasugi, Jones polynomial and classical conjectures in knot theory, Topology 26 (1987), 187-194. MR 88m:57010

[Mu2] On the braid index of alternating links, Trans. Amer. Math. Soc. 326 (1) (1991), 237-260. MR 91j:57009

[MP] and J. Przytycki, The skein polynomial of a planar star product of two links, Math. Proc. Cambridge Philos. Soc. 106(2) (1989), 273-276. MR 90f:57008

[Na] Y. Nakanishi, Unknotting numbers and knot diagrams with the minimum crossings, Math. Seminar Notes Kobe Univ. 11(2) (1983), 257-258. MR 85h:57008

[Ro] D. Rolfsen, Knots and links, Publish or Parish, 1976. MR 58:24236

[S] J. R. Stallings, Constructions of fibred knots and links, "Algebraic and geometric topology" Proc. Sympos. Pure Math., vol. 32, part 2, Amer. Math. Soc., Providence, RI, 1978, pp. 55-60. MR 80e:57004

[St] A. Stoimenow, Some examples related to 4-genera, unknotting numbers, and knot polynomials, Jour. London Math. Soc. 63(2) (2001), 487-500. MR 2002c:57011

[St2] The granny and the square tangle and the unknotting number, Topol. Appl. 117 (2002), 59-75.

[St3] - On the crossing number of positive knots and braids and braid index criteria of Jones and Morton-Williams-Franks, math.GT/0110016, Trans. Amer. Math. Soc., 354 (2002), 3927-3924.

[Th] M. B. Thistlethwaite, A spanning tree expansion for the Jones polynomial, Topology 26 (1987), 297-309. MR 88h:57007

[Th2] Kauffman's polynomial and alternating links, Topology 27(3) (1988), 311-318. MR 90c:57005

[Th3] - On the Kauffman polynomial of an adequate link, Invent. Math. 93(2) (1988), 285-296. MR 89g:57009

[Th4] - On the structure and scarcity of alternating links and tangles, J. Knot Theory Ramifications 7(7) (1998), 981-1004. MR 99k:57031

[Tr] P. Traczyk, A criterion for signed unknotting number, Contemporary Mathematics 233 (1999), 215-220. MR 2000e:57016

[Va] Quach thi Câm Vân, On a theorem on partially summing tangles by Lickorish, Math. Proc. Cambridge Philos. Soc. 93(1) (1983), 63-66. MR 84c:57003

[We] H. Wendt, Die Gordische Auflösung von Knoten, Math. Z. 42 (1937), 680-696

Department of Mathematics, University of Toronto, Ontario Canada M5S 3G3

E-mail address: stoimeno@math.toronto.edu

URL: http://www.math.toronto.edu/stoimeno/ 